\title{
GEOMETRY AND PROBABILITY IN BANACH SPACES
}

\author{
BY LAURENT SCHWARTZ
}

Introduction. The following is a brief survey of results in a circle of ideas concerned with the properties of various classes of Banach spaces (centering around the classical $L^{p}$ spaces) in terms of operators acting between them, a circle of ideas and important results which involves the names of such mathematicians as Pietsch, Maurey, H. Rosenthal, Krivine, Pisier, and others. As the title emphasizes, an important role is played by random processes with values in these Banach spaces, i.e. vector-valued probability distributions.

1. Summing maps in Banach spaces. A sequence $e=\left(e_{n}\right)_{n \in N}$ of elements of a Banach space $E$ is said to be $l^{p}$ if $\|e\|_{p}=\left(\Sigma_{n}\left\|e_{n}\right\|_{E}^{p}\right)^{1 / p}<+\infty ;\|e\|_{p}$ is the $p$-norm of this sequence (in fact, it is a norm only for $1 \leqslant p<+\infty$; we shall also work in the case $0<p \leqslant+\infty$, with the usual modifications for $p=$ $\left.+\infty:\|e\|_{+\infty}=\operatorname{Sup}_{n}\left\|e_{n}\right\|_{E}\right)$. A sequence $e$ is said to be scalarly $l^{p}$ if, for every $\xi \in E^{\prime}$ (the dual of $E$ ), the scalar sequence $\langle e, \xi\rangle=\left(\left\langle e_{n}, \xi\right\rangle\right)_{n \in N}$ is $l^{p}$, that is $\left(\Sigma_{n}\left|\left\langle e_{n}, \xi\right\rangle\right|^{p}\right)^{1 / p}<+\infty$; in this case, it can be proved (by the BanachSteinhaus theorem or the closed graph theorem) that $\|e\|_{p}^{*}=$ $\operatorname{Sup}_{\|\xi\|<1}\left(\Sigma_{n}\left|\left\langle e_{n}, \xi\right\rangle\right|^{p}\right)^{1 / p}<+\infty$; $\|e\|_{p}^{*}$ is the scalarly $l^{p}$-norm of $e$. A continuous linear map $u$ from a Banach space $E$ into a Banach space $F$ transforms trivially an $l^{p}$-sequence into an $l^{p}$-sequence, a scalarly $l^{p}$-sequence into a scalarly $l^{p}$-sequence; $u$ is said to be $p$-summing if it transforms every scalarly $l^{p}$-sequence into an $l^{p}$-sequence. By a trivial argument, if $u$ is $p$-summing, there exists a constant $C$ such that, for every sequence $e$ of $E$, the inequality $\|u(e)\|_{p} \leqslant C\|e\|_{p}^{\#}$ holds; the smallest constant $C$ is called the $p$-summing norm of $u$ and is denoted $\pi_{p}(u)$.

Every map is $(+\infty)$-summing, since $\|e\|_{+\infty}^{*}=\|e\|_{+\infty}$, and $\pi_{+\infty}(u)=\|u\|$; generally $u$ will not be better (for instance, we shall see that the identity map in an infinite-dimensional Banach space is never $p$-summing for $p<+\infty$ ). On the other hand, if $E$ is finite dimensional, a scalarly $l^{p}$-sequence is also $l^{p}$, so that every map $u$ of finite rank is $p$-summing for every $p$. A finite sum of $p$-summing maps is $p$-summing; a finite product of continuous linear maps, one of which is $p$-summing, is also $p$-summing (the $p$-summing maps "form an ideal").

Theorem (1.1) (Pietsch). For $u: E \rightarrow F$ to be $p$-summing, $p<+\infty$, it is necessary and sufficient that there exists a Radon probability $\mu$ on the unit disk

Received by the editors July 15, 1980.

1980 Mathematics Subject Classification. Primary 46B99.

Permission to reprint this article was obtained from the Southeast Asian Mathematical Society. This is a slightly modified form of the paper by Laurent Schwartz which appeared in the Special Issue, May 1979, of the SOUTHEAST ASIAN BULLETIN OF MATHEMATICS. 
$B^{\prime}$ of $E^{\prime}$, equipped with the weak-star topology $\sigma\left(E^{\prime}, E\right)$, and a constant $C$ such that, for every $x \in E$, one has

$$
\|u(x)\| \leqslant C\left(\int_{B^{\prime}}|\langle x, \xi\rangle|^{p} \mu(d \xi)\right)^{1 / p} .
$$

The best possible constant $C$ is exactly $\pi_{p}(u)$.

The proof is a fine application of the Hahn-Banach theorem and convexity.

Corollary (1.2). A p-summing map is also q-summing for $q \geqslant p$, and $\pi_{q}(u) \leqslant \pi_{q}(u)$.

Corollary (1.3) (Pietsch's factorization). (1) If $(Z, \mathscr{Z}, \mu)$ is a probability space, the canonical injection $j$ of $L^{\infty}(Z, \mathcal{Z}, \mu)$ into $L^{p}(Z, \mathcal{Z}, \mu)$ is p-summing, of $\pi$-summing norm $\leqslant 1$. Therefore every map which transmits through such a map is also p-summing.

(2) More generally, if $u: E \rightarrow F$ is a product $u_{2} u_{1}$ with the following factorization diagram

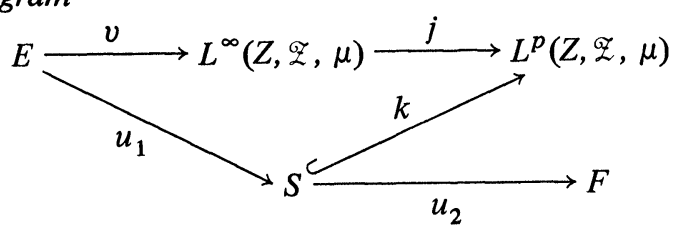

where $k$ is the canonical injection, $S$ a closed linear subspace of $L^{p}$, then $u$ is p-summing, and $\pi_{p}(u) \leqslant\left\|u_{2}\right\|\|v\|$.

(3) The converse is true: a p-summing map, $p$ finite, can be always factorized in this way, with $\pi_{p}(u)=\left\|u_{2}\right\|\|v\|$; and $Z$ can be chosen to be the unit disk $B^{\prime}$ of $E^{\prime}$, with the weak-star topology, $\mu$ Radon on $B^{\prime}$, and $v(x)(\xi)=\langle x, \xi\rangle$.

In this sense, the canonical maps of the form (1) may be considered as the prototypes of the $p$-summing maps.

Corollary (1.4). A p-summing map, p finite, is weakly compact, and transforms every weakly compact subset of $E$ into a strongly compact subset of $F$.

Proof. The canonical injection $L^{\infty} \rightarrow L^{p}$ has these properties.

COROllary (1.5). The identity map in an infinite-dimensional Banach space is never $p$-summing for $p$ finite.

Namely, if it were, the unit disk would be weakly compact, therefore strongly compact.

This can be written in another form. In an infinite-dimensional space, there always exists a sequence which is scalarly $l^{p}$ but is not $l^{p}$. As a particular case, we get Dvoretzky-Rogers' theorem: In every infinite-dimensional space, there exists a sequence which is summable but not absolutely summable (take $p=1$ in the previous result).

In some way, the characterization property (1.3) achieves the knowledge of the $p$-summing maps. But, in practice, it is never easy to recognize whether or not a map has this factorization property. For instance, when is a map 
between Hilbert spaces $p$-summing? The following theorem has no trivial proof, all the known proofs are of probabilistic nature (the "best" proofs use the Gauss probability law).

Theorem (1.6) (PeKCZINSKY). Let $u$ be a continuous map from a Hilbert space $E$ into a Hilbert space $F$. The following properties are equivalent:

(1) $u$ is Hilbert-Schmidt (i.e., for every Hilbert basis $\left(e_{i}\right)_{i \in I}$ of $E,\|u\|_{H S}=$ $\left(\Sigma_{i}\left\|u\left(e_{i}\right)\right\|_{F}^{2}\right)^{1 / 2}$ (which is independent of the basis) is finite),

(2) $u$ is $p$-summing for some finite $p$,

(3) $u$ is p-summing for every $p$,

(4) the adjoint map $u^{*}$ has one of these properties.

In these cases, $\|u\|_{H S}=\pi_{2}(u)$, and $\pi_{p}\left(u^{*}\right)=\pi_{p}(u)$ for every $p$.

The situation arising from (1.2) is the following: For a given map $u$ : $E \rightarrow F$, there exists a "summing-cut" $S(u) \in \overline{\mathbf{R}}_{+}$, such that for $p<S(u)$, $u$ is not $p$-summing, and, for $p>S(u)$, it is, and $p \mapsto \pi_{p}(u)$ is decreasing on ] $S(u),+\infty$ ]. If $S(u)=+\infty, u$ is just $(+\infty)$-summing, nothing more (see (1.5)). For $S(u)=0, u$ is $p$-summing for every $p$, and we say that $u$ is completely summing (for instance, a Hilbert-Schmidt map between Hilbert spaces is completely summing). It will be convenient to say that $u$ is $p^{+}$-summing if $r$-summing for every $r>p$ (it means $S(u)<p$ ), $p^{-}$-summing if $r$-summing for some $r<p$ (it means $S(u)<p$ ).

TheOREM (1.7). Either $S(u)=0$, or $S(u)>1$; in other words, if $u$ is $1^{-}$-summing, it is completely summing.

This property has been conjectured by Pietsch; it has been proved by Simone Chevet and Bernard Maurey in 1972. It is still often called Pietsch's conjecture, although it has been proved! We shall say that a Banach space $E$ is p-Pietsch if every $p$-summing map from $E$ into a Banach space is completely summing.

If $E$ is $p$-Pietsch, it is $q$-Pietsch for $q \leqslant p$; there exists a cut $P(E) \in \overline{\mathbf{R}}_{+}$, such that $E$ is $p$-Pietsch for $p<P(E)$, and is not for $p>P(E)$. We shall say that $E$ is $p^{+}$-Pietsch if it is $r$-Pietsch for some $r>p$ (it means $P(E)>p$ ), $p^{-}$-Pietsch if it is $r$-Pietsch for every $r<p$ (it means that $P(E) \geqslant p$ ). Theorem (1.7) expresses that every Banach space is $1^{-}$-Pietsch $(P(E)>1)$. Then

THEOREM (1.8) (Rosenthal). Let $E$ be infinite dimensional.

(1) If $E$ is $p$-Pietsch, necessarily $p<2$ (hence $1<P(E)<2$ ) and $E$ is $p^{+}$-Pietsch if $p<2$.

(2) $E$ is $p$-Pietsch, $p>1$, if and only if every map $L^{\infty} \rightarrow E$ is $p^{\prime}$-summing, $1 / p+1 / p^{\prime}=1$.

Observe that $p=1$ is surely an exception in Theorem (1.8) (2), since every map is $(+\infty)$-summing. But we may use part (1). Then $E$ is 1-Pietsch if and only if there exists some finite $r$ such that every map from $L^{\infty}$ into $E$ is $r$-summing. We shall see various instances in $\$ 2$.

The $p$-summing maps have various applications. Instead of taking sequences $e=\left(e_{n}\right)_{n \in N}$, one can take measures (the sequences are kinds of discrete measures), and study $p$-radonifying maps. Without entering into details, a $p$-radonifying map is $p$-summing; the converse is almost true, and 
true for $1<p<+\infty$. The radonifying maps give interesting tools to study stochastic processes. The continuity of the trajectories of the Brownian motion, and many other processes may be proved in this way.

2. The type and cotype of a Banach space. There are various kinds of types. We shall consider here the so-called Rademacher type. Consider a scalar random series $\Sigma_{n} \pm x_{n}$ where the $x_{n}$ are given complex numbers, and the signs \pm are random, each with probability $1 / 2$, independent of each other. An old theorem of probabilities says that this series converges almost surely if $\left(\Sigma_{n}\left|x_{n}\right|^{2}\right)^{1 / 2}<+\infty$, and diverges almost surely if $\left(\Sigma_{n}\left|x_{n}\right|^{2}\right)^{1 / 2}=+\infty$. What is the situation if we replace the numbers $x_{n}$ by vectors of a given Banach space $E$ ? The previous condition is, in general, neither necessary nor sufficient (it is necessary and sufficient iff $E$ can be renormed as a Hilbert space result of Kwapien). If $\left(\Sigma_{n}\left\|x_{n}\right\|^{2}\right)^{1 / 2}<+\infty$ implies the $\Sigma_{n} \pm x_{n}$ is almost surely convergent, we shall say that $E$ is of type 2 ; if only the stronger condition $\left(\Sigma_{n}\left\|x_{n}\right\|^{p}\right)^{1 / p}<+\infty, 0<p<2$, implies that $\Sigma_{n} \pm x_{n}$ is almost surely convergent, we shall say that $E$ is of type $p$. Of course every Banach space $E$ is of type 1 . If $E$ is of type $p$, it is of type $q$ for $q \leqslant p$; therefore there exists a cut $T(E) \in[1,2]$, such that $E$ is of type $p$ for $p<T(u)$, not of type $p$ for $p>T(u)$. $E$ will be said of type $p^{+}$if of type $r$ for some $r>p$ (it means $T(E)>p$ ), of type $p^{-}$if of type $r$ for every $r<p$ (it means $T(E)>p$ ).

A space $L^{p}, 1 \leqslant p<2$, is of type $p$, and not $p^{+}$if it is infinite dimensional; for $p \geqslant 2$ finite, it is of type 2 , but $L^{\infty}$ is only of type 1 . Type is transmitted to subspaces and factor spaces. Of course, it is possible under suitable conditions, to replace the independent random variables \pm by other ones; we don't give the results here. Also it is possible to consider a map $u: E \rightarrow F$; it is said to be of type $p$ if $\left(\Sigma_{n}\left\|x_{n}\right\|^{p}\right)^{1 / p}<+\infty$ implies that $\Sigma_{n} \pm u\left(x_{n}\right)$ converges almost surely, so that $E$ is of type $p$ iff the identity of $E$ is of type $p$. The type properties come just "below" the summing properties: one can show that a $(+\infty)_{-}$-summing map (the weakest summing property) is of type 2 (the strongest type-property); observe also that the identity map of an infinite-dimensional space cannot be $(+\infty)_{-}$-summing but can be of type 2 .

Now let us consider the converse property. $E$ is said to be of cotype $q$, $q \geqslant 2$, if the almost sure convergence of $\Sigma_{n} \pm x_{n}$ implies $\left(\Sigma_{n}\left\|x_{n}\right\|^{q}\right)^{1 / q}<$ $+\infty$ (which, for $q>2$, is weaker than $\left(\Sigma_{n}\left\|x_{n}\right\|^{2}\right)^{1 / 2}<+\infty$ ). If $E$ is of cotype $q$, it is of every cotype $r \geqslant q$, and every $E$ is of cotype $+\infty$. Therefore there exists a cut $C T(E)$, such that $E$ is of cotype $q$ for $q>C T(E)$, not of cotype $q$ for $q<C T(E)$. $E$ will be said of cotype $q^{+}$if of type $r$ for every $r>q$ (it means that $C T(E) \leqslant q$ ), of cotype $q^{-}$if of cotype $r$ for some $r<q$ (it means that $C T(E)<q$ ). A space $L^{q}$ is of cotype $q$, but not $q^{-}$if it is infinite dimensional, for $q>2$ finite, and $L^{\infty}$ is only of cotype $+\infty$; for $1 \leqslant q \leqslant 2, L^{q}$ has cotype 2 . Cotype is transmitted to subspaces, not to factor spaces; observe that every Banach space is a subspace of some space $L^{\infty}$ and a factor space of some space $L^{1}$, so that the previous observations about the types and cotypes of $L^{\infty}$ and $L^{1}$ are quite understandable. If $E$ has type $p$, then $E^{\prime}$ has cotype $p^{\prime}$, the conjugate exponent, so that, for the cuts defined previously, one has $C T\left(E^{\prime}\right) \leqslant(T(E))^{\prime}$; the converse is not true, and < 
cannot be replaced by $=$; namely, $L^{1}$ has cotype 2 , and $L^{\infty}$ is only of type 1 .

Pisier proved that $E$ is of type $1^{+}$iff $E^{\prime}$ is; there is no analogous property for exponents $p \neq 1$, as the examples of the spaces $L^{r}$ show.

Types and cotypes have a lot of applications. Let us just give some of them.

TheOREM (2.1) (MAUReY). If $u: E \rightarrow F$ is $(+\infty)_{-}$-summing, and $F$ is of cotype 2, then $u$ is 2-summing. If $u$ is 2-summing and $E$ is of cotype 2, then $u$ is completely summing (in other words, a cotype 2 is 2-Pietsch, as defined in \$1).

This explains the fact that, for Hilbert spaces, a $p$-summing map, for some $p$ finite, is completely summing; it is 2 -summing because $F$ is of cotype 2 , therefore completely summing because $E$ is of cotype 2 . Thus, this property of Hilbert spaces holds for two spaces of cotype 2.

Theorem (2.2) (Maurey). If $E^{\prime}$ is of type $p$, then $E$ is $p^{-}$-Pietsch.

This again proves Pietsch's conjecture (1.7) because $E^{\prime}$ is always of type 1 . This sufficient condition is far from being necessary; for instance $L^{1}$ is 2-Pietsch although $L^{\infty}$ is not of type 2. Maurey gave a necessary and sufficient condition in terms of type for $E^{\prime}$, but more complicated than the prior one.

It results from Theorems (2.1) and (2.2) that $L^{r}$, for $1<r<2$, is 2-Pietsch, and, for $2<r \leqslant+\infty$, is $\left(r^{\prime}\right)^{-}$-Pietsch; it is not $r^{\prime}$-Pietsch if infinite dimensional.

TheOREM (2.3) (GrothendiECK-MAUREY). Every continuous linear map from a space $L^{1}$ into a Hilbert space is completely summing.

This example, as that of (1.8) (2), is very interesting; there are spaces between which every map has some summing property. Grothendieck proved only that the map is 1-summing, and it was a very deep theorem. Now the thing is much easier; every map is 2 -summing, this part is rather easy; but $L^{1}$ has cotype 2 , therefore is 2-Pietsch, $(2.1)$, therefore every map is completely summing.

There are well-known factorization theorems for the maps between the spaces $L^{r}$, proved by Grothendieck, and extended later on; they enter into the theory of types and cotypes. Types and cotypes are also very useful to give a lot of probabilistic properties; for instance the central limit theorem and the law of iterated logarithms for scalar independent random variables may be extended to Banach random variables, provided that some properties of type or cotype are satisfied. Also observe that the partial sums of series $\Sigma_{n}^{ \pm} x_{n}$ form a martingale of a very particular kind; type and cotype may be extended to martingale-type or martingale-cotype, which are much stronger and deeper properties, studied by Pisier, related to smoothness or convexity of the disks of the Banach space.

3. Theory of finite representability and super-properties. Let $E$ and $F$ be Banach spaces. Their distance $d(E, F) \geqslant 1$ is the infimum of the $\|u\|\left\|u^{-1}\right\|$ for all the isomorphisms $u$ between $E$ and $F(d(E, F)=+\infty$ if they are not isomorphic). One says that $F$ is finitely representable into $E$ if, for every $\varepsilon>0$, every finite-dimensional subspace of $F$ has a distance $<1+\varepsilon$ with some subspace of $E$. We write then: $F$ f.r.i. $E$. If $F$ f.r.i. $E, G$ f.r.i. $F$, then $G$ f.r.i. $E$. 
A subspace of $E$ is f.r.i. $E$. Now let $P$ be a property of Banach spaces. We shall say that $E$ has super $P$ if, it not only has $P$, but also every Banach space f.r.i. $E$ has $P$. $P$ is a super-property iff super $P=P$. All these properties highly depend on the norms. If you change the norm of $E$ into an equivalent one, you change the spaces which are f.r.i. $E$ and the super-properties of $E$. If $G_{0}$ is a given Banach space, the property " $E$ is f.r.i. $G$ " is, for $E$, a super-property; Conversely, " $G_{0}$ is not f.r.i. $E$ " is, for $E$, a super-property. Reflexivity is not a super-property, and super-reflexivity has been deeply studied in the recent years; it does not depend on the norm; Per Enflo showed that $E$ is super-reflexive if and only if it can be renormed as to be uniformly convex. The space $L^{r}, 1<r<+\infty$, is super-reflexive. $E$ is super-reflexive iff $E$ is (not trivial).

As opposed to reflexivity, the following properties are super-properties: type $p$, cotype $q, p$-Pietsch. One has studied extensively the following superproperty of $E: L^{p}$ is not f.r.i. $E$. The set of numbers $p$ for which $L^{p}$ is f.r.i. $E$ is closed in $[1,+\infty]$. It is known that $L^{s}$ is a subspace of some $L^{r}$ for $1 \leqslant r \leqslant s \leqslant 2$; therefore, if $L^{s}$ is not f.r.i. $E$, then $L^{r}$ is also not for $1 \leqslant r \leqslant s \leqslant 2$. Therefore, in the interval $[1,2]$, there is a cut $(F R, \leqslant 2)(E)$, such that, for $p<(F R, \leqslant 2)(E), L^{p}$ is not f.r.i. $E$, and, for $2>p>(F R$, $\leqslant 2)(E), L^{p}$ is. There is a famous (and difficult) theorem of DvoretzkyRogers, saying that, if $E$ is infinite dimensional, $L^{2}$ is finitely representable into $E$. For $p>2$, the situation is much less known; the set of numbers $p$ for which $L^{p}$ is f.r.i. $E$ may be reduced to one point; it seems it may be an arbitrary closed subset of $] 2,+\infty]$. Then we shall define $(F R,>2)(E)$ as the largest $s \geqslant 2$ for which $L^{s}$ is f.r.i. $E$.

The Great theorem (3.1) (Maurey, Krivine, Pisier). (1) $(F R, \leqslant 2)=$ $T(E)$. In other words, $E$ has type $p^{+}$iff $L^{p}$ is not f.r.i. E. In particular, $E$ is of type $2^{-}$iff $L^{p}$ is f.r.i. E for no $p<2$.

(2) $(F R, \geqslant 2)(E)=C T(E)=(P(E))^{\prime}$ (conjugate exponent). In other words, the following properties are equivalent: $L^{r}$ is f.r.i. E for no $r>q ; E$ has cotype $q^{+} ; E$ is $\left(q^{\prime}\right)^{-}$-Pietsch.

(3) (Pisier, June 1980). Let $P(E)$ the number defined in $(1), Q(E)$ the number defined in (2), $1 \leqslant P(E) \leqslant 2 \leqslant Q(E) \leqslant+\infty$. Then, if $P(E)>1$ or $P\left(E^{\prime}\right)>$ 1 (equivalent conditions), one has $1 / P(E)+1 / Q\left(E^{\prime}\right)=1$. (If $P(E)=P\left(E^{\prime}\right)$ $=1$, the result need not hold; counterexample: if $E$ is a space $L^{\infty}, E^{\prime}$ is a space $L^{1}, P(E)=1, Q\left(E^{\prime}\right)=2$.)

This statement has been sought for during a long time; various incomplete statements have been found for years; and the proof is difficult.

Every Banach space is a subspace of some $L^{\infty}$; therefore, if $L^{\infty}$ is f.r.i. $E$, every Banach space is f.r.i. $E$. Therefore the weakest nontrivial super-property (nontrivial meaning that there is at least a Banach space which does not possess it) is $L^{\infty}$ is not f.r.i. $E$. Weakest means if $E$ has a nontrivial super-property, then it has this one. And this property is equivalent to $E$ is 1-Pietsch, or $E$ has some finite cotype. It is interesting to observe that every Banach space is $1^{-}$-Pietsch, and that the weakest super-property is to be 1-Pietsch. There are a lot of consequences of this remark: if a Banach space 
has type 1 , or if it is super-reflexive, etc., then it is 1-Pietsch. But a reflexive space (reflexivity is not a super-property) need not to be 1-Pietsch.

There are a lot of byproducts of the theorem. Consider for instance a summable sequence in $E^{\prime}$; it is known that it is not necessarily absolutely summable; but maybe it is always $l^{q}$, for some finite $q \geqslant 1$. The lowest bound of such $q$ 's is exactly $C T(E)$ (therefore $\geqslant 2$ ). In particular: if $E$ is $p$-Pietsch, every scalarly $l^{1}$-sequence is $l^{p^{\prime}}$; if every summable sequence is $l^{p^{\prime}}, E$ is $p^{-}$-Pietsch. The weakest super-property is equivalent to "there is a finite $q$ such that every summable sequence of $E$ is $l^{q}$ ".

THEOREM (3.2) (RoSENTHAL). Let $E$ be a closed vector subspace of $L^{p}$, $1 \leqslant p<2$. The following properties are equivalent.

(1) $E$ has type $p^{+}$,

(2) $L^{p}$ is not f.r.i. $E$,

(2') $E$ does not contain an isomorphic copy of $l^{p}$,

(3) every continuous linear map from $E$ into $l^{p}$ is compact,

(4) $E$ can be imbedded in some $L^{q}, q>p$,

(5) the topology of $E$ (induced by $L^{p}$ ) is equal to the topology of convergence in measure,

(6) for $p=1, E$ is reflexive.

This part of mathematics, geometry and probability in Banach spaces, is presently a very dynamic one; there are many more results than those which are given here. They have applications in probability theory, partial differential equations, harmonic analysis (lacunary series and Helson sets), and $C^{*}$-algebras.

We do not give any bibliography because all the results given here have been set forth in the Séminaires de l'Ecole Polytechnique (Séminaire Schwartz, or Séminaire Maurey-Schwartz, or Séminaire d'Analyse Fonctionnelle), of the years 1969-1970, 1972-1973, 1973-1974, 1974-1975, 1975-1976, 1977-1978, 1978-1979, 1979-1980 and these Séminaires contain an important bibliography.

Centre de Mathématiques de l'Ecole Polytechnique, Plateau de Palaiseau f-91128 Palaiseau Cedex, France 
